Rhode Island College

Digital Commons @ RIC

Master's Theses, Dissertations, Graduate

Master's Theses, Dissertations, Graduate

Research and Major Papers Overview

Research and Major Papers

2017

\title{
Neck Circumference and Mallampati Classification
}

Patrick B. Brennan

Follow this and additional works at: https://digitalcommons.ric.edu/etd

Part of the Other Nursing Commons

\section{Recommended Citation}

Brennan, Patrick B., "Neck Circumference and Mallampati Classification" (2017). Master's Theses, Dissertations, Graduate Research and Major Papers Overview. 253.

https://digitalcommons.ric.edu/etd/253

This Major Paper is brought to you for free and open access by the Master's Theses, Dissertations, Graduate Research and Major Papers at Digital Commons @ RIC. It has been accepted for inclusion in Master's Theses, Dissertations, Graduate Research and Major Papers Overview by an authorized administrator of Digital Commons @ RIC. For more information, please contact digitalcommons@ric.edu. 
Neck Circumference and Mallampati Classification

by

Patrick B. Brennan

A Major Paper Submitted in Partial Fulfillment

of the Requirements for the Degree of

Master of Science in Nursing

in

The School of Nursing

Rhode Island College

2017 


\begin{abstract}
Difficult and failed intubations contribute to morbidly and mortality in anesthesia practice. Mallampati is one of the most widely used airway assessments but research shows it is highly variable in its ability to predict difficult intubation. Neck circumference is an objective assessment not commonly used, but has been shown to assess the degree of difficulty with intubation. Following Institutional Review Board (IRB) approval from both Charter Care and Rhode Island College, utilizing a descriptive design, 23 subjects had their neck circumference measured and Mallampati class assessed. The results revealed a relationship between increased neck circumference and Mallampati classification. The average neck circumference of the 23 subjects was $40.35 \mathrm{~cm}$ with an average Mallampati class of 2.54. The average neck circumference for Mallampati class 1 was $39.3 \mathrm{~cm}$ versus $42.8 \mathrm{~cm}$ for Mallampati class 4. Planning for a difficult airway and the assessments to use are highly variable. Practitioners pull from personal experiences as well as their training when it comes to how they provide care. Research has found that neck circumference is an objective assessment that correlates to difficult intubations. Mallampati is one of the standard airway assessments despite research showing that it does not accurately predict intubation difficulty. However, additional research is needed to understand the role neck circumference plays in everyday practice.
\end{abstract}




\section{Table of Contents}

Background/Statement of the Problem ..................................................................... 2

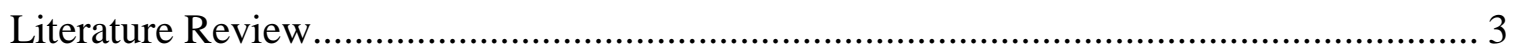

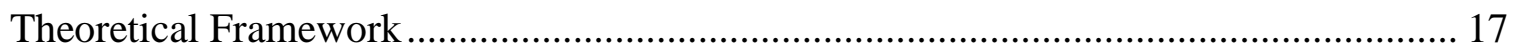

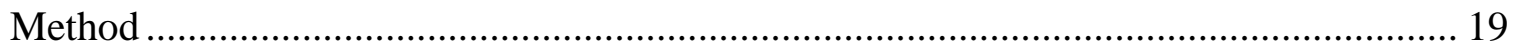

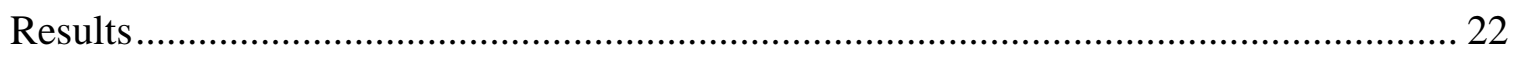

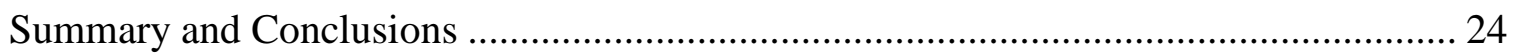

Recommendations and Implications for Advanced Nursing Practice .......................... 27

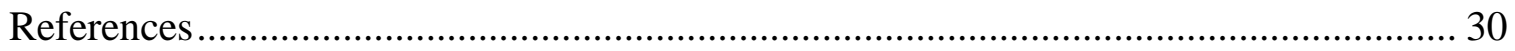

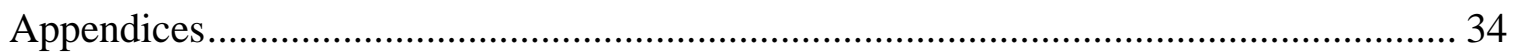


Neck Circumference and Mallampati Classification

\section{Background/Statement of the Problem}

The profession of anesthesia is grounded in a scientific knowledge base with emphasis on physiology and anatomy. Anesthesia providers are considered airway experts. However, as is true within every discipline, practitioners tend to demonstrate individual variations in the way they assess and care for patients rather than consistently using an evidence-based approach. Difficult and failed intubations contribute to morbidity and mortality in anesthetic practice (Liu, Yi, Guo, Ma, \& Huang, 2016). The need for a thorough and accurate airway assessment is pivotal to patient safety. Early recognition of a potentially difficult airway would allow the anesthesia provider time to prepare appropriately for a difficult intubation.

There are multiple, different airway assessments that can be completed, with one of the most common being Mallampati. The Mallampati classification, which is widely used, is subject to interpretation of the clinician completing the assessment. Mallampati assessment is completed by having the patient open their mouth and assigning a classification based on what the practitioner visualizes (Gupta, Sharma, \& Jain, 2005). In contrast, neck circumference is an objective measurement that has been show to correlate with difficult intubation. The purpose of this study was to examine the relationship between Mallampati classification and neck circumference.

Next, the review of the literature will be presented. 


\section{Literature Review}

The literature was reviewed by using both PubMed and google scholar. Search terms used in a variety of combinations included Mallampati, neck circumference, obesity, anesthesia, intubation, difficult intubation, airway assessment, and surgery. Literature dating back to 2002 were included in the review of the literature. Research focused primarily outside the operating room or without anesthesia were excluded. Although obesity was a focus because of anatomical changes to the airway that impact intubation, research focusing on non-obese patients was also included to ensure completeness.

\section{Obesity}

The World Health Organization (WHO, 2015) described obesity and overweight as abnormal or excessive fat accumulations that may impair health. Worldwide obesity is on the rise, doubling since 1980 (WHO). The WHO classifies obesity as a body mass index of greater than or equal to 30 and overweight as a body mass index greater than or equal to 25 . Concern about ever-increasing body mass indexes has lead adiposity to be included in the global non-communicable disease targets, with a goal of halting it by 2025 (Non- Communicable Disease [NCD] Risk Factor Classification, 2016).

In 2014, 1.9 billion adults worldwide were overweight, with 600 million considered obese (WHO, 2015). Globally, 13\% of the worlds' adult population is obese, with slightly more women than men (WHO, 2015). According to the NCD Risk Factor Classification (2016), men from wealthy English speaking countries and women from central Latin America had the highest increase in BMI. Obesity was once considered a wealthy countries' problem. However, energy dense foods that are 
high in fat are becoming readily available at ever decreasing prices. Changes in work and modes of transportation are shifting populations away from physical labor to one of a sedentary lifestyle. Lack of support from health sectors, education, food processing and marketing are all contributing to an ever-growing problem (WHO).

The impact income and race have on obesity effect men and women differently. Obesity decreases as income decreases among non-Hispanic black and Mexican American men (Ogden, Lamb, Carroll, \& Flegal, 2010). Obesity increased by $40.8 \%-44.5 \%$ among non-Hispanic black and Mexican American men with incomes at or above the poverty level (Ogden et al.). There was not, however, a statically relevant difference in obesity when considering income among nonHispanic white men (Ogden et al.). Twenty-nine percent of women living in households at or above the poverty line are obese and $42 \%$ of women living in households with incomes 130\% below the poverty line are obese (Ogden et al.). NonHispanic white men and women regardless of income make up the majority of the obese population in the United States (US) (Ogden et al.).

Obesity is not just a change in body weight, but a disease state that has a multitude of complications. In 2008, obesity was associated with an annual medical cost of 147 billion dollars in the US (CDC, 2015). Medical expenses increased an average of $\$ 1,429$ higher than non-obese patients in the US (CDC). Cardiovascular complications like heart failure or myocardial infarction and stroke accompany obesity, both of which were leading causes of death in 2012 (WHO, 2015). Obese and overweight individuals are at increased risk for diabetes, musculoskeletal changes such as osteoarthritis, and a variety of cancers (WHO). Childhood obesity is also 
associated with increased risk of obesity, disability, and premature death in adulthood (WHO).

\section{Obesity-Associated Comorbidities Negatively Impacting Anesthesia}

Outcomes. Perioperative care of obese patients is becoming common as the prevalence of obesity continues to increase globally and especially in the US. Ultimately the goal of anesthesia is to provide care for obese patients while minimizing risks associated with comorbidities. The US is home to $13 \%$ of the world's obese population (Ortiz \& Kwo, 2015). Obesity affects almost every body system and can result in vastly different clinical presentations, which makes treating the obese patient challenging. In a study completed by Lindauer, Steurer, Müller, \& Dullenkopf (2014), 182 patients undergoing bariatric procedures were followed over two years. Among these patients, common histories included diabetes, gastroesophageal reflux disease, EKG changes, impaired left ventricular function and tobacco use.

Major cardiovascular changes occur as the body attempts to adapt to increase body mass and metabolic demand (Ortiz \& Kwo, 2015). Thirty-one percent of individuals with long-standing obesity will develop structural and functional changes of the heart. Mechanical impairment occurs from structural changes to the heart driven by direct cardiotoxic effects from insulin resistance, neurohumoral overactivation, and nocturnal hypoxia and hypercarbia. Coronary artery disease is often missed in obese patients because symptoms such as dyspnea and chest pain are common to the population. Risk factors for coronary artery disease include diabetes, 
hypertension and dyslipidemia, all of which are common among obese patients (Ortiz \& Kwo).

Monteiro \& Azevedo (2010) discussed the impact metabolic syndrome has on the obese patient. Metabolic syndrome is a cluster of disorders consisting of glucose intolerance, central obesity, hypertension, dyslipidemia and a decrease in high-density lipoprotein. Metabolic syndrome puts the patient at increased risk for developing coronary artery disease, type 2 diabetes and cancer. An overall inflammatory response is common in the obese patient with metabolic syndrome. The inflammatory response in obese patients occurs in the absence of injury or infection, unlike an inflammatory response in a healthy adult. Overall metabolic stress leads to organelle dysfunction on a cellular level involving the mitochondria and endoplasmic reticulum. The endoplasmic reticulum plays an important role in lipid, protein, cholesterol, and glucose metabolism. The cluster of disorders associated with metabolic syndrome can make performing a safe anesthetic a challenge. Glucose intolerance puts the patient at risk for both hypoglycemia and hyperglycemia, necessitating close glucose monitor during the intraoperative period. Hypertension is associated with damage to multiple organ systems requiring precise blood pressure control (Monteiro \& Azevedo).

As an individuals' BMI continues to increase, especially above 45 , there is a greater impact to the respiratory system (Ortiz \& Kwo, 2015). Pharyngeal structures change and increase in size and are prone to collapse as weight increases. Obstructive sleep apnea effects between $40-90 \%$ of obese individuals. Individuals with obstructive sleep apnea are prone to cardiopulmonary complications such as atrial 
fibrillation, respiratory failure, emergency intubation, non-invasive and mechanical ventilation. Severe asthma that responds poorly to conventional treatments like inhaled corticosteroids is more likely to occur in obese patients. Pulmonary hypertension is also common among obese patients and puts them at risk for developing congestive heart failure, respiratory failure, hemodynamic instability, and sepsis (Ortiz \& Kwo).

Along with cardiovascular and respiratory changes, endocrine, musculoskeletal, gastrointestinal and psychiatric disorders occur. Patients with BMIs greater than 40 are seven times more likely to have diabetes, putting them at risk for poor wound healing and renal failure (Ortiz \& Kwo, 2015). It is estimated that $91 \%$ of patients scheduled for weight loss surgery have non-alcoholic fatty liver disease (NAFLD). Anesthetic drug consideration is of utmost importance because NAFLD effects hepatic enzymes involved in drug metabolism. Physiologic changes place obese patients at increased risk for gastroesophageal reflux disease (GERD). Gastroesophageal reflux disease increases the risk of developing ulcers and adenocarcinoma. Mental health is also an issue with the obese population having approximately a $25 \%$ increase in mood and anxiety disorders (Ortiz \& Kwo).

\section{Anesthesia, Intubation, and Difficult Intubation}

Airway management is a key piece of the anesthesia provider's role.

Anesthetized patients without a secured airway via endotracheal tube or laryngeal mask airway lose protective reflexes and have relaxation of tissues that put them at risk for obstruction (Flood, Rathmell, \& Shafer, 2015). Successfully intubating the anesthetized patient can be difficult at times but is a key component to safe 
anesthesia. Difficult and failed intubations account for considerable morbidity and mortality in anesthetic practice (Liu et al., 2016). Obesity is associated with a variety of comorbidities requiring increased vigilance when planning an intubation. Novice anesthetists are more likely to encounter difficult or prolonged intubation times leading to adverse outcomes (Liu et al.).

Traditional tracheal intubation is done under direct laryngoscopy using either a Macintosh or Miller blade (Liu et al, 2016). In a study completed by Liu et al., intubations on patients with normal appearing airways by the novice anesthetist using either direct laryngoscopy or a video assisted device were compared. Video laryngoscopy allows for a potentially better view of the glottis than direct laryngoscopy. One hundred eighty-two patients were randomly assigned to nine anesthetists to be intubated with either video laryngoscopy or direct laryngoscopy with a Macintosh blade. Evaluation was completed by comparing time until intubation and the degree of ease of intubation. Of the 182 patients in the study, two had failed intubations using video laryngoscopy and one with direct laryngoscopy. Time until successful intubation was slightly longer in the group using video laryngoscopy requiring 30.6 seconds versus 28.7 seconds with a Macintosh. Ease of intubation was assessed using a five-point scale, one being the easiest, and 5 being the most difficult. Novice anesthetists found the use of video laryngoscopy to be easier than direct laryngoscopy, rating it as one to two versus two to three. (Liu et al., 2016). Regardless of the technique used, intubation is a skill that requires ample training to be successful. 
The American Society of Anesthesiologist (ASA) released an updated version of their difficult airway algorithm in 2013 (Apfelbaum et al., 2013). According to the ASA there is no standard definition of difficult intubation but rather a complex interaction between patient, clinical setting and skills of the provider. The ASA guidelines for difficult intubation are in place to reduce the likelihood of adverse outcomes. A thorough airway assessment must be completed on all patients under the care of anesthesia providers. The difficult airway algorithm guides providers in their decision-making process when a difficult intubation is suspected. A copy of the ASA difficult airway algorithm can be found in Appendix A.

Anesthesia providers have varying levels of education, training and experience. Each provider may have different skill sets but are individually held to the same standards of care. Crosby (2011) discussed whether there is a role for clinical practice guidelines in airway management. Although airway complications are rare and most intubations are successful with direct laryngoscopy, outcomes for patients of failed intubations are severe (Crosby). Novice anesthetists are introduced to strategies for handling difficult airways but proper technique comes with experience (Crosby). Crosby opined that poor patient outcomes from difficult intubation could be avoided with proper training. Algorithms for intubation are in place but only work if the anesthetist is willing to utilize them. In a major study looking at the use of an airway algorithm, intubation was successful in 95 of 100 cases (Combes; cited in Crosby, 2011).

In the study completed by Combes et al. (2004), 41 senior anesthesiologists went through two months of training on the use of airway algorithms. Following the 
training the subjects were asked to strictly adhere to the airway algorithm over the two-year study period. Difficult intubation was defined as two failed attempts with the laryngoscope being removed from the patients mouth between attempts. Steps in the algorithm ranged from mask ventilation or the use of a laryngeal mask airway to tracheostomy with jet ventilation. A total of 11,257 intubations were performed during the study period with 100 cases of unexpected difficult airways. Deviation from the algorithm occurred in 3 of the 100 cases of unexpected difficult airway. The remaining participants were successfully intubated using the algorithm. A total of 80 patients were intubated using a gum elastic bougie with the remaining patients being ventilated using a laryngeal mask airway. Combes et al. found that adherence to a simple difficult airway algorithm was efficacious in the management of patients when an unanticipated difficult airway arose.

Planning and thorough assessment is important for every patient encounter regardless of how challenging the provider deems the intubation to be. In a study completed by Vasconcelos et al. (2014), 87 patients' records were reviewed to see if key components were completed prior to intubation. Data collected included whether a Mallampati classification was assigned, if fasting was assessed, if equipment was ready and if the endotracheal tube placement was verified by auscultation or capnography. Eight-seven percent of the patients did not have a Mallampati assessment performed. Fasting was assessed in only $50 \%$ of the patients. Ninety-five percent of the time endotracheal tube placement was verified using capnography, with auscultation being assessed $28 \%$ of the time. Individual variations in how practitioners provide care play a significant role in overall patient outcomes. 


\section{Difficult Intubation and Relationship to Obesity}

The anatomical and physiologic changes that take place with obesity make intubation more difficult. According to De Jong et al. (2014), difficult intubation is defined as three or more attempts to achieve tracheal intubation or intubations requiring more than 10 minutes. Complications associated with difficult intubation range from esophageal intubation, dental damage, severe hypoxemia, to cardiovascular collapse (De Jong et al.). Obesity is often associated with a variety of comorbidities leaving the patient with poor reserve for handling physiologic stress.

Difficult intubations can be challenging to predict in the obese patient. In a study completed by Ezri et al. (2003), 50 obese patients underwent ultrasound of the neck to quantify difficult intubation with anatomical changes. Findings suggested that difficult intubation was associated with increase neck circumference as well as increases in soft tissue. Nine of the 50 patients included were deemed a difficult intubation; of the nine, six had a Mallampati classification of two and three had a classification of three. The mean neck circumference was 50 centimeters versus 43.5 centimeters for the easy intubation group. Ultrasound of different areas of the neck showed soft tissue to be between 25 - 33 millimeters for the difficult intubation group versus 17.5 - 27.4 millimeters for the easy intubation group (Erzi et al.). Standard airway assessments do not assess the amount of soft tissue present in the obese patient's airway.

In a study completed by De Jong et al. (2014), difficult intubation and its associated complications were assessed in both the intensive care unit and the operating room. Fourteen hundred intubations in the intensive care unit and 11,035 
intubations in the operating room over a period of years were involved in the study. Obese patients accounted for $20 \%$ of the intensive care unit cohort and $19 \%$ of the operating room cohort. De Jong et al. found that difficult intubations took place more often in the intensive care unit. Difficult intubation accounted for $8 \%$ of the obese patients in the operating room in contrast to $16 \%$ of the obese patients in the intensive care unit. Factors that contributed to difficult intubation in obese patients were Mallampati scores, limited mouth opening, reduced mobility of the cervical spine, and obstructive sleep apnea (De Jong et al.).

Changes to the obese patient's airway involve excess fat tissue in the velopalate, retropharynx and submandibular regions making intubation difficult (Langeron, Birenbaum, Sache, \& Raux, 2014). Obesity is also associated with a decrease pulmonary functional residual capacity, making obese patients prone to desaturation during the intubation process. Atelectasis in dependent portions of the lungs is common in the obese patient putting them at greater risk for poor oxygenation (Langeron et al.). The combination of both anatomical and physiological changes to the obese patient put them at risk for complications during the intubation process.

Adverse outcomes during the intubation process arise from a variety of factors including situational stress, low familiarity with other tracheal intubation techniques and lack of adherence to published difficult airway algorithms (Borges et al., 2010). Using high fidelity simulation, Borges et al. investigated adherence to published airway algorithms in "cannot intubate" and "cannot ventilate" situations. A total of 38 anesthesiologists were invited to participate in various simulations. Participants were 
presented with an hour-long debriefing following the first simulation and discussed in detail the ASA difficult airway algorithm. Results showed that 75\% $(n=38)$ of participants had at least one major deviation for the algorithm. Borges et al. stated that experienced anesthesiologist used their current skills and knowledge to manage an airway emergency and modified the algorithm to their comfort level.

\section{Measures to Assess Intubation Risk: Mallampati and Neck Circumference}

A thorough airway assessment is a standard of practice for anesthesia providers. Mallampati classifications are one of the more common airway assessments completed. Accurate Mallampati classifications are contingent on the participation of the patient, the lack of phonation during the exam and the training of the anesthesia provider. Variation in assigned Mallampati classification can take place necessitating two examinations (Gupta et al., 2005). Neck circumference, which is not widely used, has shown to be correlated with difficult intubation: there is a correlation between increasing circumference and difficult intubation (Gonzalez et al., 2008). Neck circumference, measured from the same anatomical landmarks, is an easy and objective airway assessment, unlike Mallampati.

The Mallampati classification is a common airway assessment completed by anesthesia providers, the measurement component of which includes a subjective component. Mallampati classification is completed by having the patient open his/her mouth and comparing tongue size to pharyngeal size (Gupta et al., 2005). Patients are classified as Mallampati one to four based on what the examiner visualizes. Mallampati class one means the examiner can see the soft and hard palate, the full uvula, as well as the anterior and posterior pillars. Mallampati class four means the 
examiner can see only the hard palate and no other structures. False positives are common, resulting in lower classifications, especially if the patient phonates during the exam (Gupta et al.).

Neck circumference is a relatively new measure slowly being incorporated into daily practice. Multiple studies have attempted to assess the role neck circumference plays in relation to airway assessment. Studies have used both the thyroid cartilage and cricoid cartilage as landmarks for neck measurement. Consistency with measurement is important to maintain the accuracy of the data. Using the chosen anatomical landmark, the patients' neck is measured from that point completely around using a tape measure.

In a study completed by Brodsky, Lemmens, Brock-Utne, Vierra, and Saidman (2002), 100 patients undergoing elective surgery were assessed with a number of airway techniques including Mallampati, neck circumference, mouth opening and thyromental distance. Higher Mallampati classifications would alert the anesthesia provider to the possibility of difficult intubation. However, the study results demonstrated how variable Mallampati results can be, with higher classifications not always correlating to difficult intubation. Of the 88 patients deemed easy intubations, 25 had a Mallampati class of 3, and 1 had a class of 4 . None of the 12 patients deemed problematic intubations had a Mallampati class of 4, with all being classified as 2 or 3 (Brodsky et al.).

The average weight of the subjects participating in the study was 124.8 kilograms with an average neck circumference of 50.5 centimeters. Results demonstrated that as neck circumference increased so did the possibility of difficult 
intubation. Larger neck circumference was associated with a poor view during direct laryngoscopy. One failed intubation took place after three attempts with direct laryngoscopy. The patient was a 38-year-old male, who weighed 141 kilograms, with a neck circumference of 58 centimeters, and a Mallampati classification of three. Brodsky et al. (2002) found the single best indicator of difficult intubation to be neck circumference.

Neck circumference was compared to a variety of other airway assessment techniques in 70 obese and 61 lean patients (Gonzalez et al., 2008). Average BMI for the obese group was 44 compared to 24 for the lean group. Twenty-three patients in the obese group had Mallampati classifications of 3-4 compared to three patients in the lean group. Average neck circumference for the obese group was 42 centimeters verses 39 centimeters for the lean group. Twelve of the 131 patients were deemed difficult intubations; $67 \%$ had a Mallampati class greater than 3, compared to $13 \%$ of the easy intubation group. Mean neck circumference of the difficult intubation group was 47 centimeters compared to 40 centimeters for the easy intubation group (Gonzalez et al.).

In a study completed by Magalhães, Marques, Govêia, Ladeira, and Lagares (2013), 83 patients were divided into two groups (obese and non-obese) and assessed using various airway techniques. The obese group consisted of 43 patients with an average neck circumference of 40.7 centimeters. Thirty-five of the obese patients had Mallampati classifications of one to two and six patients had classifications of three to four. The non-obese group consisted of 45 patients with an average neck circumference of 36.4 centimeters. Forty-four of the non-obese patients had 
Mallampati classifications of one to two and one patient had a classification of three to four (Magalhães et al., 2013). Results showed that both neck circumference and Mallampati scores were higher in the obese group.

Next, the theoretical framework used to guide this study will be presented. 


\section{Theoretical Framework}

The Neuman Systems Model, a client-focused framework, was chosen to guide this research (Appendix B). A systems approach is utilized to assess stressors effecting the client (Neuman \& Fawcett, 2002). The model attempts to consider all variables that may impose actual or possible stressors and how system stability is achieved. All systems inherently have structure and dynamic organization, principles and laws and terms effecting the constraints of the environment. For change to happen, there is a complex relationship between the need to retain valued elements from the past and the need for flexibility to allow new structure to emerge (Neuman $\&$ Fawcett).

The Neumans Systems Model is based on wholeness, wellness, client perception and motivation, dynamic systems perspective and interactions with the environment to mitigate harm from internal and external stressors (Neuman \& Fawcett, 2002). The variables that can impact the client can be physiological, psychological, sociocultural, developmental and spiritual. According to Neumann \& Fawcett, the client is viewed as the system. The central core (Appendix B) consists of basic survival factors such as genetic features and strengths and weaknesses of the system (Neuman \& Fawcett).

Surrounding the central core are flexible and normal lines of defense as well as lines of resistance. The flexible line of defense acts as the initial barrier preventing stressors from reaching the central core (Neuman \& Fawcett, 2002). Simple or multiple stressors can rapidly reduce the effectiveness of the flexible line of defense. Working toward the central core, the next barrier is the normal line of defense. The normal line of defense represents what the client has become and their normal state of 
wellness. Once the normal line of defense is breached, the stressor can reach the client. Finally, the last defense to the central core are the lines of resistance. The lines of resistance contain known and unknown internal and external resources that support the client's response to stress (Neuman \& Fawcett).

The Neuman Systems Model also encompasses primary, secondary and tertiary health promotion and wellness. Primary prevention happens before a stressor has reached the client, secondary prevention takes place after a stressor has reached the client, and finally tertiary prevention happens following treatment of a stressor reaction. For the purposes of this study, the focus will be on secondary prevention utilizing the Neuman Systems Model. Secondary prevention goals are wellness attainment by strengthening the internal lines of defense. The goal is to provide client specific interventions, contributing to system stability, and minimize stressors effecting the client.

The Neuman systems model was utilized with a focus on secondary health promotion, representing airway assessment utilizing neck circumference in this study. This could be useful in identifying potentially at risk patients to avoid adverse outcomes.

Next, study methods will be presented. 


\section{Method}

\section{Purpose}

The purpose of this study was to examine the relationship between Mallampati classification and neck circumference. Mallampati classification is routinely assigned to all preoperative patients during the anesthesia history and physical. Neck circumference, measured in centimeters, is not routinely used but research has shown increasing circumference correlates with difficult airways. Trends between both Mallampati classification and neck circumference were assessed.

\section{Design}

The study employed a descriptive design. Mallampati classification and neck circumference were collected at Pre-Admission Testing (PAT) by the researcher.

\section{Sample}

Only those patients who underwent PATs at the data collection site were included in the study. Inclusion criteria included: adult patients greater than 18 years old; those requiring PATs; patients undergoing surgery at the main clinical site; and ambulatory or inpatient status. Exclusion criteria included: pediatric patients; those who cannot sign consent; non-English speaking patients; and patients who undergo phone PATs. A sample of 23 subjects was recruited for the study. Subjects included were chosen based on convenience and those willing to participate were involved. Site

The primary site of data collection was a community hospital in Rhode Island.

\section{Procedures}

Prior to data collection, IRB approval from both Rhode Island College and Charter Care was obtained. Permission was obtained from the Director of Saint 
Joseph's Hospital School of Nurse Anesthesia and the Director of Anesthesia for Our Lady of Fatima Hospital.

PAT Data Collection Procedures. Patients who require a degree of closer monitoring before surgery undergo pre-admission testing (PAT). This allows providers a chance to obtain lab work, EKGs, health histories, and other important information days to weeks before the patient's procedure. The PAT procedures are completed by staff nurses at the main clinical site on varying days throughout the week.

Prior to data collection, the Chief of the Anesthesia department was informed of the days chosen for data collection. The researcher first reviewed the PAT list to initially screen for potential eligibility for the study. After identifying potentially eligible subjects, the researcher then approached patients in PAT to explain the purpose of the study and describe to potential subjects what would be involved if they decided to participate. Interested subjects had the consent reviewed and explained and questions answered by the researcher. Those who agreed to participate were asked to sign the consent document. .

Next, neck circumference and a Mallampati Classification was determined. Neck circumference measurement was completed by using the thyroid cartilage as a land mark. Utilizing the thyroid cartilage as the level of measurement allowed for consistent data collection on all subjects. Mallampati classification was determined by having the patient open their mouth and assigning a class based on what can be visualized. Those subjects who phonated during the assessment of Mallampati were asked to open their mouths a second time without phonating for accuracy. The score 
recorded was the one without phonation. Data was recorded on a data collection sheet developed by this researcher (Appendix C).

\section{Measurement}

Neck circumference and Mallampati classification were collected as previously described. No other data was collected.

\section{Data Analysis}

Once the data were collected, Mallampati classification and neck circumference were assessed and compared.

Next, the results will be presented. 


\section{Results}

A total of 23 subjects met the inclusion criteria and were interviewed. Table 1 illustrates participants' Mallampati score and neck circumference.

Table 1

Participants' Mallampati Score as Compared to Neck Circumference $(N=23)$

\begin{tabular}{|c|c|}
\hline Mallampati Score & Neck Circumference $(\mathbf{c m})$ \\
\hline 1 & 43.5 \\
\hline 1 & 45 \\
\hline 1 & 41.5 \\
\hline 1 & 33.5 \\
\hline 1 & 33 \\
\hline 2 & 37 \\
\hline 2 & 41 \\
\hline 2 & 37.5 \\
\hline 2 & 38.5 \\
\hline 2 & 43.5 \\
\hline 2 & 36 \\
\hline 2 & 36 \\
\hline 2 & 39 \\
\hline 3 & 41 \\
\hline 3 & 47 \\
\hline 3 & 45 \\
\hline 3 & 41.5 \\
\hline 3 & 34.5 \\
\hline 3 & 41 \\
\hline 3 & 49 \\
\hline 4 & 44.5 \\
\hline 4 & 39.5 \\
\hline 4 & 44.5 \\
\hline
\end{tabular}


Table 1 illustrates the wide spread in neck circumference measurements for each of the corresponding Mallampati scores. The largest neck circumference measurement was 49 $\mathrm{cm}$ with a Mallampati score of 3 . More subjects had a Mallampati score of $2(\mathrm{n}=8)$ and 3 $(n=7)$ and fewer had Mallampati scores of $1(n=5)$ or $4(n=3)$.

Table 2 below illustrates the mean, median and mode neck circumference by Mallampati classification.

Table 2

Mean, median, and mode neck circumference measurements (cm) by Mallampati score

\begin{tabular}{|l|l|l|l|}
\hline Mallampati score & \multicolumn{1}{|c|}{$\begin{array}{c}\text { Mean neck } \\
\text { circumference (cm) }\end{array}$} & $\begin{array}{c}\text { Median neck } \\
\text { circumference (cm) }\end{array}$ & $\begin{array}{c}\text { Mode neck } \\
\text { circumference (cm) }\end{array}$ \\
\hline $\begin{array}{l}\text { Mallampati (1-4) } \\
\text { (N=23) }\end{array}$ & 40.54 & 41 & 41 \\
\hline $\begin{array}{l}\text { Mallampati 1 } \\
(\mathbf{n}=5)\end{array}$ & 39.3 & 41.5 & NA \\
\hline $\begin{array}{l}\text { Mallampati 2 } \\
(\mathbf{n = 8})\end{array}$ & 38.56 & 38 & 36 \\
\hline $\begin{array}{l}\text { Mallampati 3 } \\
(\mathbf{n = 7})\end{array}$ & 42.71 & 41.5 & 41 \\
\hline $\begin{array}{l}\text { Mallampati 4 } \\
(\mathbf{n = 3})\end{array}$ & 42.83 & 44.5 & 44.5 \\
\hline
\end{tabular}

Mean neck circumference ranged from $38.56-42.71 \mathrm{~cm}$. The largest mean neck circumference was $42.71 \mathrm{~cm}$ and corresponded to subjects with a Mallampati score of 3 $(n=7)$.

Next, the summary and conclusions will be presented. 


\section{Summary and Conclusions}

Anesthesia providers are considered airway experts and routinely instrument patients' airways for various surgical procedures. Failed intubations add to morbidly and mortality in anesthesia (Liu et al., 2016) and there are various airway assessments that can be completed to assess intubation difficulty. However, no single assessment has been accepted as the gold standard in anesthesia practice. Neck circumference and Mallampati assessment are used by anesthesia providers to determine intubation difficulty. The Mallampati assessment is a subjective assessment that is currently more widely used by practitioners but does not always accurately assess the degree of difficulty involved with intubation (Gupta et al., 2005). Neck circumference is an objective airway assessment that has been shown to accurately identify the risk for difficult intubations (Gonzalez et al., 2008). The purpose of this study was to examine the relationship between Mallampati classification and neck circumference. The Newman's Systems Model (Neuman \& Fawcett, 2002) was utilized as the framework to guide the study, with a focus on the secondary health promotion aspect of the model.

Institutional review board (IRB) approval was obtained from the primary site of data collection as well as Rhode Island College. Over a three-day period, 23 subjects were identified that met the inclusion criteria and consented to participate in the study. Prior to obtaining consent, the study was explained to subjects in simple terms, including risks and benefits, as well as the right to refuse. When the consent document was signed by the participant, the study procedures were implemented.

Mallampati scores were collected by having subjects open their mouths and then a score was assigned based on the anatomical landmarks viewed. Subjects tended to 
phonate when prompted to open their mouths which alters the Mallampati score. A second assessment was completed on those subjects who phonated with repeat instruction on the exam to ensure appropriate scoring was taking place. The second exam without phonation was the score recorded. Neck circumference was obtained by using disposable tape measures and using the thyroid cartilage as a landmark.

The average neck circumference for all four Mallampati classes was $40.54 \mathrm{~cm}$ and the average Mallampati score was 2.35. The mean neck circumferences $(\mathrm{cm})$ for each Mallampati class (1-4) were $39.3,38.56,42.71$, and 42.83 respectively. The data suggest a relationship between increase neck circumference and higher Mallampati class. The average neck circumference for Mallampati four was $42.83 \mathrm{~cm}$. Neck circumferences for patients who had a Mallampati class of one ranged from $33-43.5 \mathrm{~cm}$. The largest neck circumference recorded was $49 \mathrm{~cm}$ and the subject had a Mallampati class of three. Although the sample size was small there was a relationship between larger neck circumferences and higher Mallampati scores.

From the data collected, it appears that both neck circumference and Mallampati score increase proportionately together. However, the overall sample size was small and body weight was not collected from participating subjects. According to Brodsky et al. (2002), in a study of 100 subjects, neck circumference was a better indicator of difficult intubation with the average weight of participants. Weight would have been a pertinent third data point to collect in this study; it might also have been useful to collect other data including height, gender and age. 
Another limitation was the small sample size. A larger sample size with more widespread Mallampati scores would have provider more information. Personal bias during data collection might have occurred. Ideally, the individual collecting the data would have been blind to what the studies purpose was. Variations between Mallampati scores are based on small anatomical changes and bias could have impacted the scoring. The study was further limited by the convenience sampling used.

There is a solid body of literature that demonstrates that Mallampati classification is highly variable. Neck circumference measurement is objective and has been shown to accurately predict difficult intubations, although similar studies completed showed no direct correlation between both airway assessments (Brodsky et al., 2002). Neck circumference, unlike Mallampati score, is not widely used in daily practice. As a more objective measure that is also quick, easy to complete, and not as subject to individual variation, it is recommended that the neck circumference assessment be integrated into practice as an adjuvant assessment measure.

Next, recommendations and implications for advanced practice nursing will be presented. 


\section{Recommendations and Implications for Advanced Nursing Practice}

Advanced practice registered nurses play an integral role in shaping current

evidence based health care practices. Certified registered nurse anesthetists (CRNAs) perform airway assessments every day on every patient they encounter. However, variations in the way each practitioner assesses and manages patients generally evolves from individual experiences as well as how the practice setting functions. At a minimum, an airway assessment, most commonly the Mallampati classification, must be completed on each patient.

Shifting from what has been traditionally done can be difficult for providers who have become comfortable with their practice. It is important for everyone, but especially new practitioners, to bring evidence based research into daily practice. Prior research demonstrated that increasing neck circumference correlated with difficult intubations (Brodsky et al., 2002). Current providers can safely incorporate neck circumference into situations where a patient is deemed a potential difficult intubation. Providers should be encouraged to use a variety of airway assessments and not succumb to using Mallampati as the sole airway assessment.

Intubations are not just facilitated in an operating room setting by an anesthesia provider. For example, both emergency room and critical care providers care for patients requiring mechanical ventilation and airway instrumentation. These providers may not have the opportunity to develop airway assessment skills in their respective work settings. Certified registered nurse anesthetists can act as leaders and educators for other APRNs who are working in a setting where having the ability to intubate is an important skill. Providing education on objective airway assessments, like neck circumference, can 
give providers not as experienced in airway assessments an easy reliable tool to use. Not only can CRNAs incorporate neck circumference into their daily practice but they can also educate other APRNs on its use.

Neck circumference is a measurement that could be completed on patients if the practitioner is worried about their airway but it may not alter their plan. Anesthesia providers use multiple assessments at the same time when planning an intubation technique and they use the tools they are most experienced with to secure a difficult airway. An experienced provider is already formulating a plan the minute they lay eyes on the individual they will be anesthetizing. Neck circumference would be useful for a new practitioner when planning an anesthetic. In conjunction with other airway assessments, it could be a useful objective tool to prepare for airway instrumentation.

The need for an accurate airway assessment is key to decreasing morbidity and mortality associated with intubation. Policies exist to maintain a standard of care and ultimately protect the public. However, individual training and experience is different for each provider. Allowing practitioners to practice utilizing evidence based research and pulling from their own personal experience requires balance. Anesthesia practice would suffer in a setting that forced providers to practice based all on policy or all on personal experience. Protocols and guidelines, such as the ASA difficult airway algorithm, give providers an established tool they can utilize to guide them through challenging patient encounters.

Additional research on the role neck circumference plays in anesthesia is key to understanding when and if it should be used. Other key factors that should be included in 
future research include obesity, sex, height, age, as well as existing comorbid conditions. A successful provider is one who includes policy, evidence based research, and personal experience into the care they provide patients every day. Further research is needed to understand the role neck circumference plays in daily practice and to answer such questions as whether to utilize neck circumference on every patient or just those who have a comorbidity that would make intubation difficult. 


\section{References}

Adult Obesity Facts. (2015, September 21). Centers for Disease Control and

Prevention. Retrieved June 1, 2016, from

https://www.cdc.gov/obesity/data/adult.html

Apfelbaum, J. L., Hagberg, C. A., Caplan, R. A., Blitt, C. D., Connis, R. T., Nickinovich, D. G., ... \& Guidry, O. F. (2013). Practice guidelines for management of the difficult airway: An Updated Report by the American Society of Anesthesiologists Task Force on Management of the Difficult Airway. The Journal of the American Society of Anesthesiologists, 118(2), 251-270.

Borges, B. C., Boet, S., Siu, L. W., Bruppacher, H. R., Naik, V. N., Riem, N., \& Joo, H. S. (2010). Incomplete adherence to the ASA difficult airway algorithm is unchanged after a high-fidelity simulation session. Canadian Journal of Anesthesia/Journal canadien d'anesthésie, 57(7), 644-649.

Brodsky, J. B., Lemmens, H. J., Brock-Utne, J. G., Vierra, M., \& Saidman, L. J. (2002). Morbid obesity and tracheal intubation. Anesthesia \& Analgesia, $94(3), 732-736$.

Combes, X., Le Roux, B., Suen, P., Dumerat, M., Motamed, C., Sauvat, S.... \& Dhonneur, G. (2004). Unanticipated difficult airway in anesthetized patient's prospective validation of a management algorithm. The Journal of the American Society of Anesthesiologists, 100(5), 1146-1150.

Crosby, E. T. (2011). An evidence-based approach to airway management: is there a role for clinical practice guidelines? Anaesthesia, 66(s2), 112-118. 
De Jong, A., Molinari, N., Pouzeratte, Y., Verzilli, D., Chanques, G., Jung, B., ... \& Jaber, S. (2014). Difficult intubation in obese patients: incidence, risk factors, and complications in the operating theatre and in intensive care units. British Journal of Anaesthesia, aeu373.

Ezri, T., Gewürtz, G., Sessler, D. I., Medalion, B., Szmuk, P., Hagberg, C., \& Susmallian, S. (2003). Prediction of difficult laryngoscopy in obese patients by ultrasound quantification of anterior neck soft tissue. Anaesthesia, 58(11), $1111-1114$.

Frerk, C., Mitchell, V. S., McNarry, A. F., Mendonca, C., Bhagrath, R., Patel, A., \& Ahmad, I. (2015). Difficult Airway Society 2015 guidelines for management of unanticipated difficult intubation in adults. British Journal of Anaesthesia, 115(6), 827-848.

Gonzalez, H., Minville, V., Delanoue, K., Mazerolles, M., Concina, D., \& Fourcade, O. (2008). The importance of increased neck circumference to intubation difficulties in obese patients. Anesthesia \& Analgesia, 106(4), 1132-1136.

Gupta, S., Sharma, R., \& Jain, D. (2005). Airway assessment: predictors of difficult airway. Indian J Anaesth, 49(4), 257-262.

Langeron, O., Birenbaum, A. L. S. F., Le Saché, F., \& Raux, M. (2014). Airway management in obese patient. Minerva anestesiologica, 80(3), 382-392.

Lindauer, B., Steurer, M. P., Müller, M. K., \& Dullenkopf, A. (2014). Anesthetic management of patients undergoing bariatric surgery: two year experience in a single institution in Switzerland. BMC Anesthesiology, 14(1), 125. 
Liu, Z. J., Yi, J., Guo, W. J., Ma, C., \& Huang, Y. G. (2016). Comparison of McGrath Series 3 and macintosh laryngoscopes for tracheal intubation in patients with normal airway by inexperienced anesthetists: A randomized study. Medicine, 95(2).

Magalhães, E., Marques, F. O., Govêia, C. S., Ladeira, L. C. A., \& Lagares, J. (2013). Use of simple clinical predictors on preoperative diagnosis of difficult endotracheal intubation in obese patients. Revista Brasileira de Anestesiologia, 63(3), 262-266.

Monteiro, R., \& Azevedo, I. (2010, July 14). Chronic Inflammation in Obesity and the Metabolic Syndrome. Retrieved November 22, 2017, from https://www.hindawi.com/journals/mi/2010/289645/

Neuman, B. M., \& Fawcett, J. (2002). The Neuman systems model. Pearson Education, Incorporated.

Non-communicable diseases risk factor collaboration. (2016). Trends in adult bodymass index in 200 countries from 1975 to 2014: a pooled analysis of 1698 population-based measurement studies with 19.2 million participants. The Lancet, 387(10026), 1377-1396.

Obesity and overweight. (n.d) (2015, January). World Health Organization. Retrieved June 10, 2016, from http://www.who.int/mediacentre/factsheets/fs311/en/

Ogden, C. L., Lamb, M. M., Carroll, M. D., \& Flegal, K. M. (2010). Obesity and socioeconomic status in adults: United States, 2005-2008. NCHS data brief, $50(51), 1-8$. 
Ortiz, V. E., \& Kwo, J. (2015). Obesity: physiologic changes and implications for preoperative management. BMC Anesthesiology, 15(1), 97.

Shafer, S., Rathmell, J. P., \& Flood, P. (2015). Stoelting's pharmacology and physiology in anesthetic practice (5th ed.). Philadelphia, PA: Lippincott, Williams, \& Wilkins.

Vasconcelos, H., Bomfim, C. C., Mello, M. J. G. D. M., Borges, P. S. G. N., Couceiro, T. C. D. M., \& Orange, F. A. D. (2014). Is the anesthesiologist actually prepared for loss of airway or respiratory function? A cross-sectional study conducted in a tertiary hospital. Revista da Associação Médica Brasileira, 60(1), 40-46. 


\section{Appendix A \\ American Society of Anesthesiologists ${ }^{\circ}$ \\ DIFFICULT AIRWAY ALGORITHM}

1. Assess the likelihood and clinical impact of basic management problems:

- Difficulty with patient cooperation or consent

- Difficult mask ventilation

- Difficult supraglottic airway placement

- Difficult laryngoscopy

- Difficult intubation

- Difficult surgical airway access

2. Actively pursue opportunities to deliver supplemental oxygen throughout the process of difficult airway management.

3. Consider the relative merits and feasibility of basic management choices:

- Awake intubation vs. intubation after induction of general anesthesia

- Non-invasive technique vs. invasive techniques for the initial approach to intubation

- Video-assisted laryngoscopy as an initial approach to intubation

- Preservation vs. ablation of spontaneous ventilation

4. Develop primary and alternative strategies:
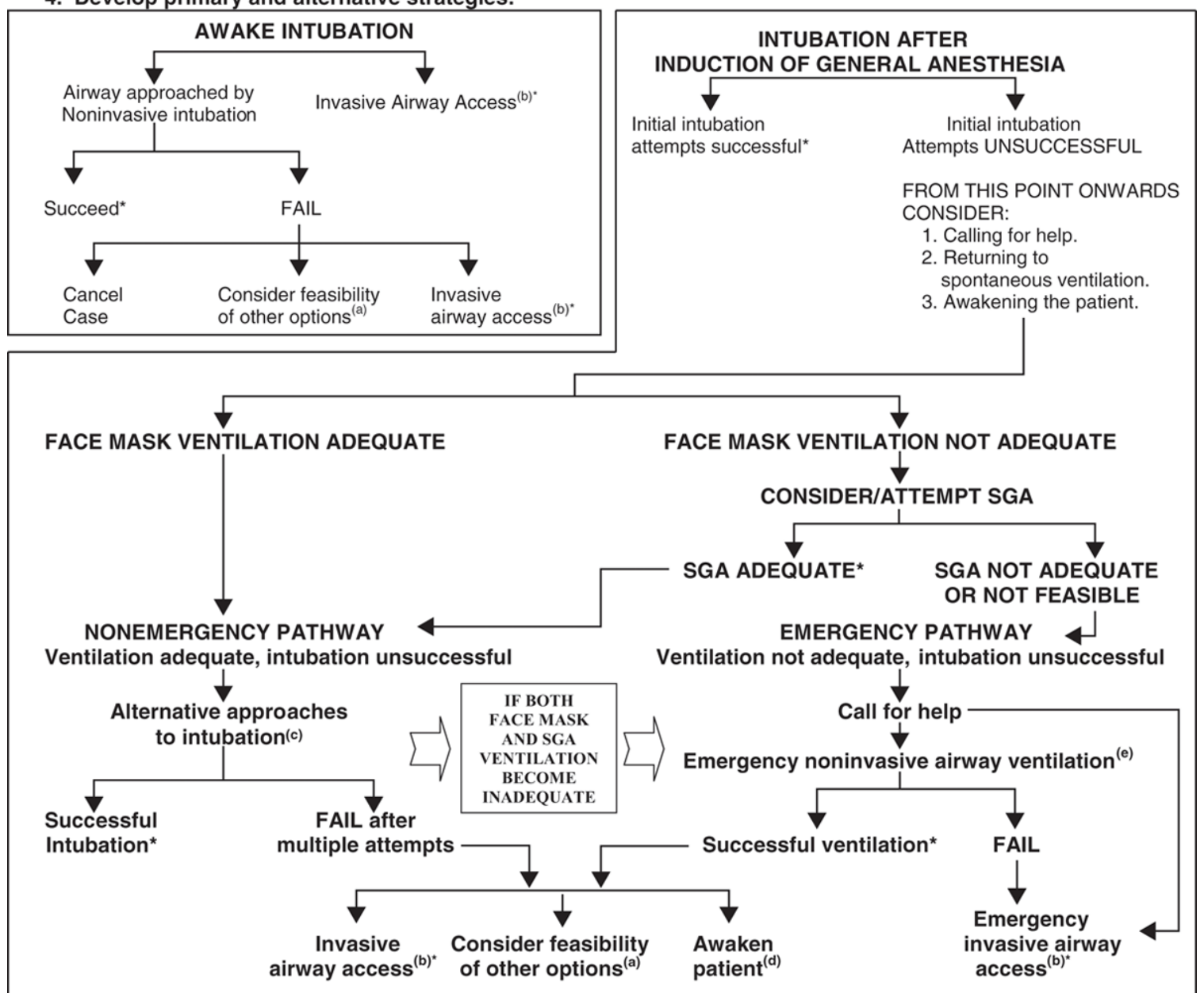


\section{Appendix B}

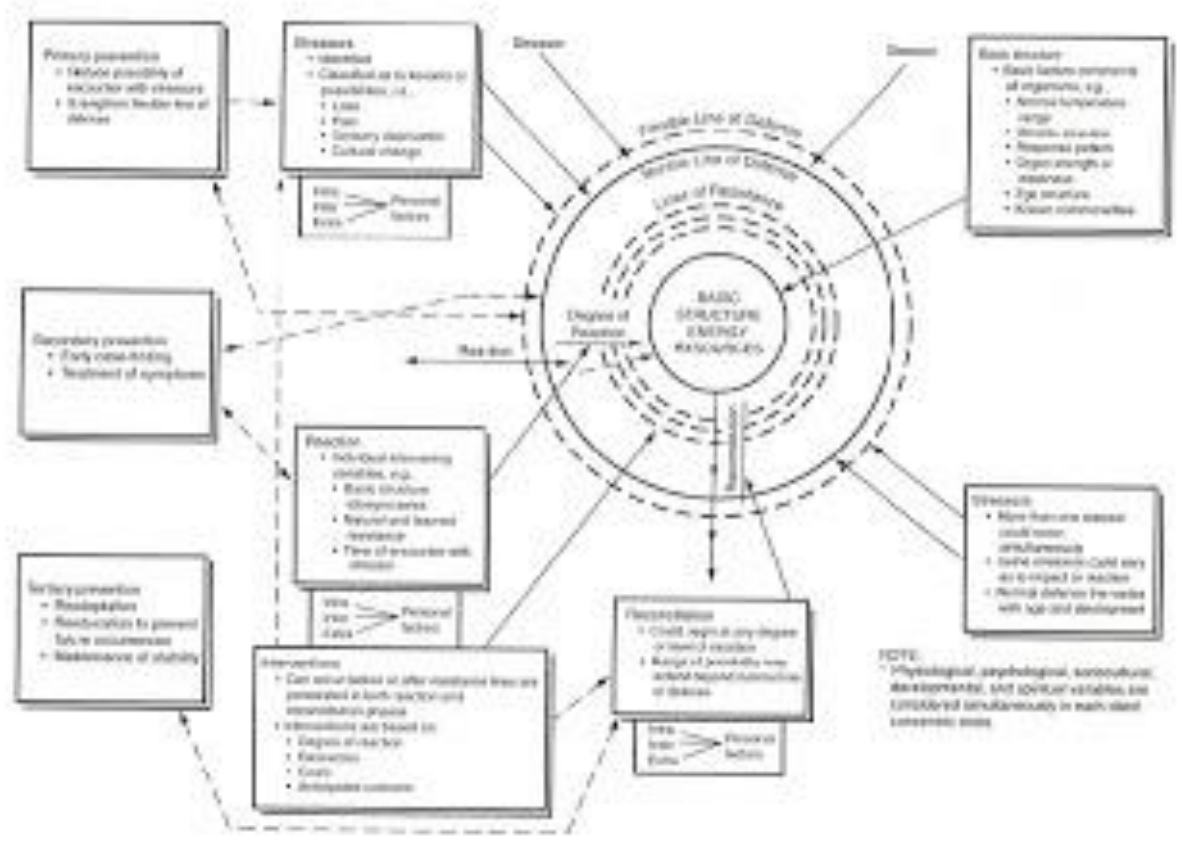

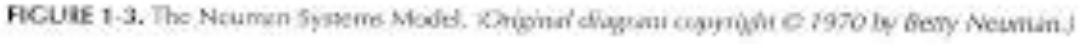


Appendix C

Data Collection Sheet

\begin{tabular}{|l|l|}
\hline Mallampati & $\begin{array}{c}\text { Neck } \\
\text { Circumference }\end{array}$ \\
\hline . & \\
\hline & \\
\hline & \\
\hline & \\
\hline & \\
\hline
\end{tabular}

